\title{
Leishmaniose visceral em um canino de Cruz Alta, Rio Grande do Sul, Brasil
}

\author{
Visceral leishmaniasis in a dog in Cruz Alta, Rio Grande do Sul, south Brazil
}

\author{
Cristina Krauspenhar ${ }^{I}$ Cristiane Beck ${ }^{\text {II }}$ Vitor Sperotto ${ }^{\text {II }}$ Aline Alves da Silva ${ }^{\text {II }}$ \\ Rodrigo Bastos $^{\text {II }}$ Lauro Rodrigues ${ }^{\text {II }}$
} \begin{abstract}
um canino, macho, Akita, cinco anos, que foi atendido no Hospital Veterinário da Universidade de Cruz Alta, RS, após residir três anos no Estado de Alagoas. Clinicamente observouse emagrecimento progressivo, lesões crostosas na ponta das orelhas, focinho e região periorbital, úlcera na região distal do membro torácico direito, opacidade da córnea, vômitos e eventuais episódios de diarréia. O exame sorológico para leishmaniose pelo método de ELISA resultou positivo. Na avaliação da medula óssea por imprints (corados por Panóptico), foi possível observar formas amastigotas no citoplasma de macrófagos. A histopatologia consistia de reação linfohistioplasmocitária acentuada, com estruturas basofílicas sugestivas de formas amastigotas de Leishmania sp. no citoplasma de macrófagos. A aplicação da técnica de imunoistoquímica mostrou imunomarcação positiva para Leishmania $\mathrm{sp}$.
\end{abstract}

Palavras-chave: Leishmaniose visceral, Leishmania sp., amastigotas, ELISA, imunoistoquímica.

\section{ABSTRACT}

A case of visceral leishmaniasis in a five-year-old, intact male Akita presented to the Veterinary Hospital at the University of Cruz Alta, RS, Brazil after having lived in the state of Alagoas for three years is described. Clinical signs included progressive weight loss, crusty lesions on the tip of the ears, nose and periorbital region, an ulcer at the distal region of the right forelimb, corneal opacity, vomiting, and occasional bouts of diarrhea. Serologic testing for leishmaniasis by ELISA was positive. Macrophages with intracytoplasmic amastigote forms were observed in bone marrow imprints stained with Diff Quick.

\begin{abstract}
Histopathology was characterized by marked lymphohistioplasmacytic inflammation associated with intrahistiocytic basophilic structures suggestive of leishmanial amastigotes. The diagnosis of leishmaniasis was confirmed by positive immunohistochemical staining for Leishmania sp..
\end{abstract}

Key words: Visceral leishmaniasis, Leishmania sp., amastigotes, ELISA, immunohistochemistry.

A leishmaniose visceral (LV)é uma zoonose causada pelo protozoário intracelular do gênero Leishmania (KEENAN et al., 1984). A doença é endêmica nas regiões Norte, Nordeste, Centro-Oeste e Sudeste (LUVIZOTTO et al., 2005), tendo como agente uma única espécie do subgênero Leishmania (Leishmania) chagasi (TAFURI et al., 2001), que é transmitida ao hospedeiro vertebrado através da picada de um inseto hematófago Lutzomyia longipalpis (ASHFORD, 1998; SLAPPENDEL \& FERRER, 1998).

A espécie canina apresenta alta prevalência da infecção, sendo um reservatório importante, pois alberga o parasita na derme, reforçando a importância da espécie canina no ciclo biológico da doença, atuando como fonte de infecção para os flebotomínios durante o repasto sangüíneo (MATTOS et al., 2004; LUVIZOTTO et al., 2005). Após alimentar-se no hospedeiro vertebrado, o inseto ingere macrófagos e monócitos infectados contendo amastigotas de

ISetor de Patologia, Universidade de Cruz Alta (UNICRUZ), 98025-810, Cruz Alta, RS, Brasil. E-mail: ckrauspenhar@yahoo.com.br. Autor para correspondência.

IISetor de Clínica de Pequenos Animais, UNICRUZ, Cruz Alta, RS, Brasil. 
Leishmania spp. No intestino do vetor, as amastigotas irão transformar-se em promastigotas. Estas formas são injetadas na pele do hospedeiro durante a alimentação do vetor, transformam-se em amastigotas e irão multiplicar-se no interior de macrófagos. Dividem-se por divisão binária, rompem o macrófago e infectam novas células (AEBISCHER, 1994; ASHFORD, 1998).

Os animais apresentam febre, perda de peso progressiva, anemia, alopecia localizada ou generalizada, ulcerações da pele (especialmente no focinho, na orelha e nas extremidades), conjuntivite, ceratite, ornicogrifose, linfoadenopatia e presença de nódulos que eventualmente podem ulcerar (KOUTINAS et al., 1999; MOZOS et al., 1999; FEITOSA et al., 2000).

O objetivo deste relato é descrever os aspectos clínicos e patológicos de um canino, macho, Akita, cinco anos, que foi atendido no Hospital Veterinário da Universidade de Cruz Alta, RS, após residir três anos no Estado de Alagoas. Clinicamente observou-se emagrecimento progressivo, lesões crostosas na ponta das orelhas, no focinho, na região periorbital e lesão focal ulcerativa na pele do membro pélvico direito (Figura 1), opacidade da córnea, vômitos e eventuais episódios de diarréia. Foram coletadas amostras de sangue para hemograma, que resultou em eosinofilia e anemia e também para realização do Teste de ELISA. O animal foi submetido à eutanásia e necropsiado no Setor de Patologia da Universidade de Cruz Alta.
As lesões macroscópicas consistiam de leve icterícia, linfadenopatia acentuada, esplenomegalia, hepatomegalia com acentuação do padrão lobular. Os rins estavam acentuadamente pálidos e a medula óssea intensamente avermelhada. Na avaliação da medula óssea por imprints (corados por Panóptico) realizados durante a necropsia, foi possível observar formas amastigotas no citoplasma de macrófagos (Figura 2), os quais eventualmente estavam rompidos. A análise microscópica do linfonodo, do baço, da medula óssea, do fígado, do rim, da pele e do coração, corados com H\&E, apresentaram reação linfohistioplasmocitária acentuada. Numerosos macrófagos reativos, contendo vacúolos parasitóforos intracitoplasmáticos com estruturas basofílicas sugestivas de formas amastigotas de Leishmania sp., foram observados na medula óssea, no fígado, no baço e no linfonodo. A aplicação da técnica de imunoistoquímica, utilizando anticorpo primário e KIT LSDA, mostrou imunomarcação positiva para Leishmania sp. na medula óssea, no fígado, no linfonodo e no rim (Figura 3).

A leishmaniose visceral é uma zoonose com distribuição mundial, sendo que a doença é descrita em todas as regiões do Brasil, exceto na região Sul (BRASIL, 2003). Entretanto, POCAI et al. (1998) descrevem cinco casos de leishmaniose visceral na região de Santa Maria, Rio Grande do Sul, sugerindo a existência de reservatório deste parasita e do vetor artrópode na região. No entanto, após notificação e informações da Secretaria de Saúde, não foram

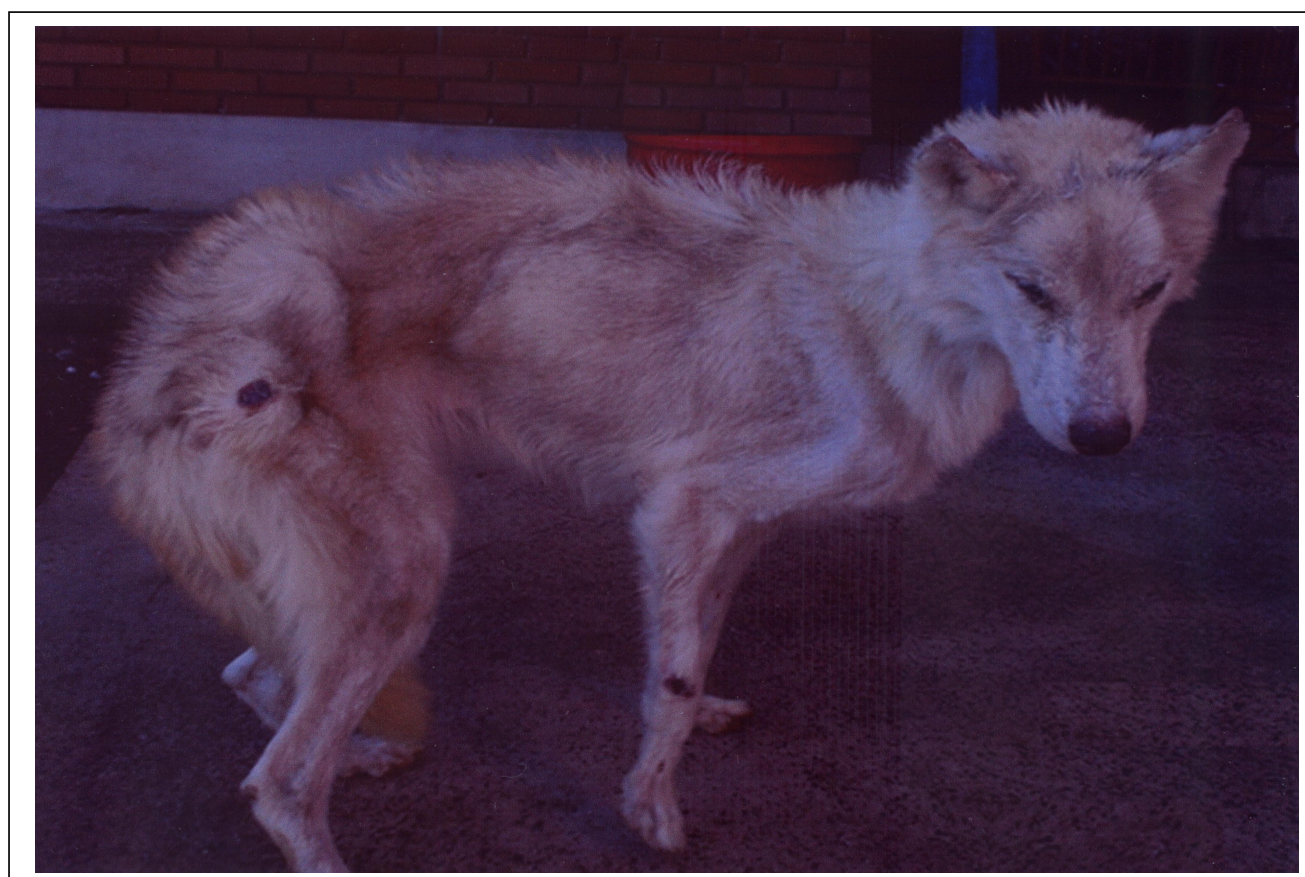

Figura 1 - Leishmaniose visceral canina. Emagrecimento progressivo, lesões crostosas na ponta das orelhas, focinho, região periorbital e lesão focal ulcerativa na pele do membro pélvico direito. 


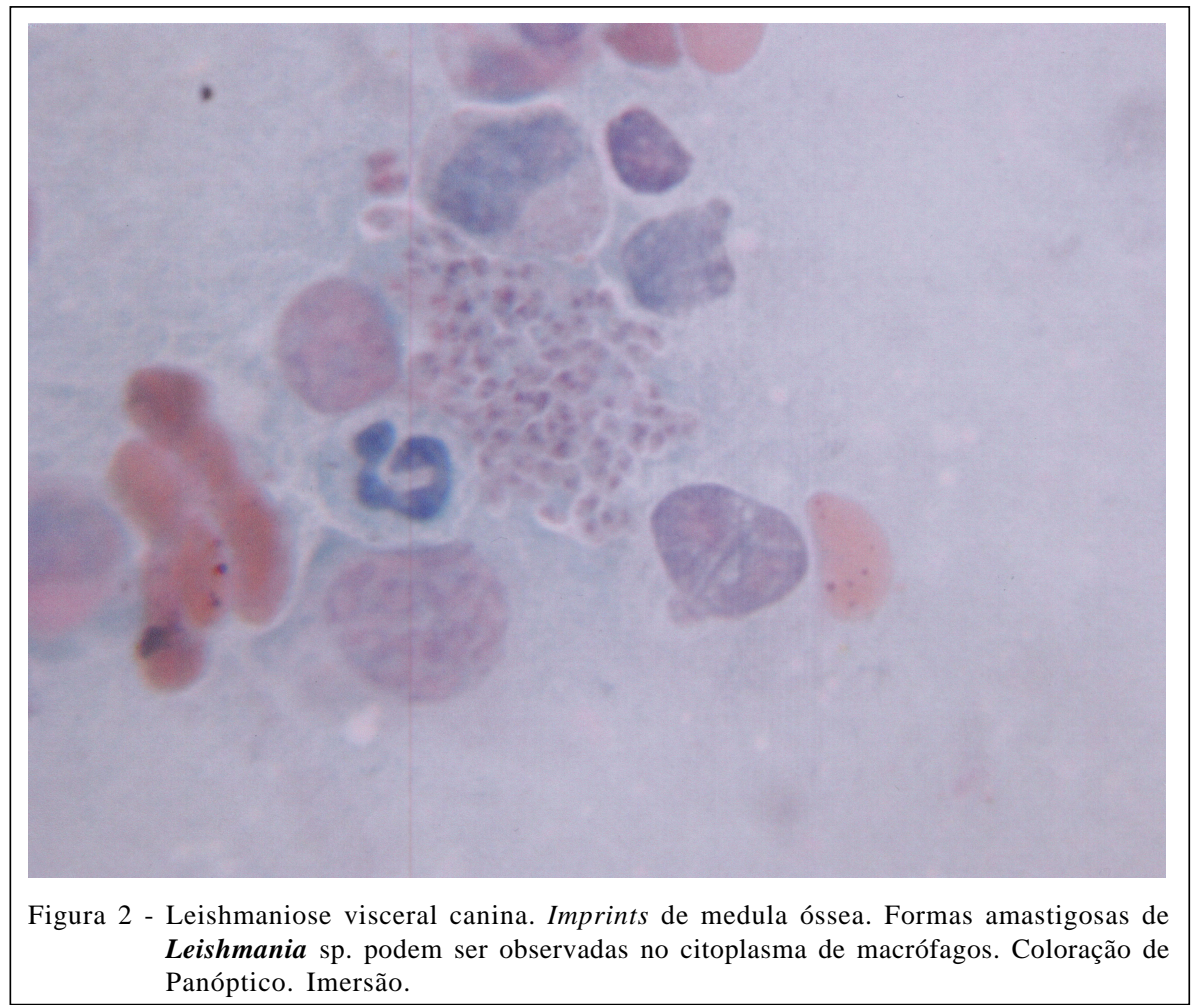

encontrados exemplares do vetor na região de Santa Maria e de Cruz Alta, sugerindo a interrupção do ciclo do parasita. Assim, nas áreas não-endêmicas, a enfermidade ocorre em cães importados, freqüentemente após vários anos (BRASIL, 2003), como observado neste caso.
Para o diagnóstico da leishmaniose, a presença do parasita constitui requisito básico, mas os métodos sorológicos, como o Ensaio imunoenzimático (ELISA), são úteis para a triagem de casos (MOURA et al., 1999). No caso relatado, foram observadas formas amastigotas em imprints de medula

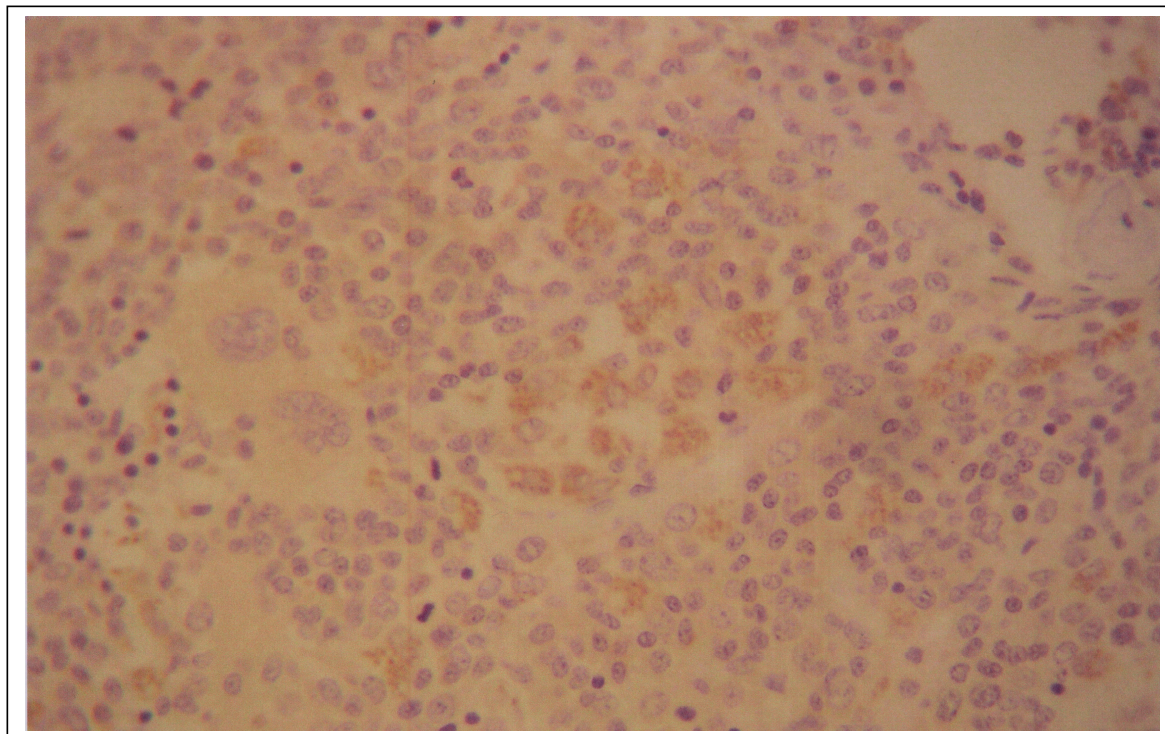

Figura 3 - Leishmaniose visceral canina. Imunoistoquímica de medula óssea. Imunomarcação positiva no citoplasma de macrófagos contendo numerosas formas amastigosas. KIT LSDA, obj.40. 
óssea, e, segundo alguns autores, a demonstração de amastigotas em aspirados ou imprints, corados por Giemsa, Wright e Leishman, fornecem diagnóstico definitivo(SANTAROSA\& OLIVEIRA, 1997; NELSON \& COUTO, 2001).

O diagnóstico de leishmaniose nas regiões endêmicas é realizado por testes sorológicos (POCAI et al., 1998). Entretanto, neste caso, o canino adoeceu em área indene, apesar de ter se infectado no Estado de Alagoas, onde a doença é endêmica e emergente. No caso aqui descrito, o diagnóstico foi clínico, através de teste sorológico (ELISA) e pós-morte, baseando-se nas lesões encontradas em vários órgãos. A confirmação do diagnóstico foi através de técnica imunoistoquímica.

Normalmente os casos de leishmaniose canina precedem os casos humanos, já que os cães são os principais reservatórios domésticos e fundamentais na manutenção do ciclo da doença (SANTA ROSA \& OLIVEIRA, 1997). No entanto, isso não significa que haverá casos em humanos, já que, por tratar-se de uma área indene, não foram observados vetores na região, fato que sugere a interrupção do ciclo da parasita. Entretanto, a ocorrência de um caso esporádico de leishmaniose visceral canina serve para alertar os clínicos da importância dessa doença, a qual vem emergindo no Brasil.

\section{AGRADECIMENTOS}

Os autores agradecem a Dra. Maria Cecília Rui Luvizotto, da Universidade Estadual Paulista (UNESP), pela realização da técnica de imunoistoquímica.

\section{REFERÊNCIAS}

AEBISCHER, T. Recurrent cutaneous leishmaniasis: A role for persistent parasites? Parasitology Today, v.10, p.25-28, 1994.

ASHFORD, R.W. The leishmaniases. In: PALMER, S.R. et al. Zoonoses. Oxford: Oxford University, 1998. Cap.43, p.527543.
BRASIL. Ministério da Saúde. Departamento de Vigilância Epidemiológica. Manual de Vigilância e controle da leishmaniose visceral. Brasília, 2003. 122p.

FEITOSA, M.M. et al. Aspectos clínicos de cães com leishmaniose visceral no município de Araçatuba São Paulo (Brasil). Clínica Veterinária, Ano 5, n.28, p.36-44, 2000.

KEENAN, C.M. et al. Visceral leishmaniasis in the German Shepherd dog. I. Infection, clinical disease, and clinical pathology. Veterinary Pathology, v.21, p.74-79, 1984.

KOUTINAS, A.F. et al. Clinical considerations on canine visceral leishmaniasis in Greece: a retrospective study of 158 cases (1989-1996). J Am Anim Hosp Assoc, v.35, p.376383, 1999.

LUVIZOTTO, M.C.R. et al. Lesão nodular na cavidade oral de cão causada por Leishmania sp. - relato de casos. Arq Bras Méd Vet Zootec, v.57, p.18-19, 2005.

MATTOS, Jr.D.G. et al. Aspectos clínicos e de laboratório de cães soropositivos para leishmaniose. Arq Bras Méd Vet Zootec, v.56, n.1, p.199-122, 2004.

MOURA, S.T. et al. Diagnóstico de leishmaniose canina na área urbana do município de Cuiabá, estado de Mato Grosso, Brasil. Braz J Vet Res Anim Sci, v.36, n.2, p.123-126, 1999.

MOZOS, E. et al. Leishmaniosis and generalized demodicosis in three dogs: a clinicopathological and immuno-histochemical study. J Comp Path, v.120, p. 257-268, 1999.

NELSON, R.W.; COUTO, C.G. Medicina interna veterinária de pequenos animais. 2.ed. Rio de Janeiro: Guanabarra Koogan, 2001. p.1037.

POCAI, E.A. et al. Leishmaniose visceral (calazar). Cinco casos em cães de Santa Maria, Rio Grande do Sul, Brasil. Ciência Rural. v.28, n.3, p.501-505, 1998.

SANTA ROSA, I.C.A.; OLIVEIRA, I.C.S. Leishmaniose visceral: breve revisão sobre uma zoonose reemergente. Clínica veterinária, ano II, n.11, p.24-28, 1997.

SLAPPENDEL, R.J.; FERRER, L. Leishmaniasis. In: GREENE, C.E. Infectious diseases of the dog ad cat. 2.ed. Philadelphia: Saunders, 1998. Cap.73, p.450-458.

TAFURI, W.L. et al. Canine visceral leishmaniose: a remarkable histopathological picture of one case reported from Brazil. Veterinary Parasitology, v.96, p.203-212, 2001. 